CORRESPONDENCE

\title{
Profile of capillary-leak syndrome in patients received chimeric antigen receptor $\mathrm{T}$ cell therapy
}

(c) The Author(s), under exclusive licence to Springer Nature Limited 2022

Bone Marrow Transplantation (2022) 57:661-663; https://doi.org/ 10.1038/s41409-022-01562-4

\section{TO THE EDITOR:}

Autologous T cells modified to express a recombinant chimeric antigen receptor (CAR-T) for tumor-related antigens has demonstrated exciting efficacy in treating hematological malignancies as well as other diseases [1-3]. Until now the US Food and Drug Administration has approved five products commercially and there are growing numbers of clinical trials being conducted around the world. Besides the promising outcomes of the novel treatment, the toxicities followed by CAR-T therapy are the main barrier to its application. The most frequent and described toxicities are cytokine release syndrome (CRS), which is a hyperimmune inflammatory response caused by the rapid increase in excessive cytokines as results of activation and proliferation of CAR-T cells. The clinical manifestations of CRS present as fever, fatigue, edema, acute kidney injury, cardiac toxicity, capillary-leak syndrome (CLS) and other symptoms of systemic inflammatory response syndrome [4-6]. As a syndrome of the severe CRS, CLS is characterized by profound vascular leakage, resulting in the transfer of protein-rich fluid from the intravascular to the interstitium. On the one hand, it leads to insufficient vascular content and reduced effective circulating blood volume, which can manifest clinically as hypotension, low central venous pressure, blood concentration, hypoproteinemia, shock and acute kidney injury; on the other hand, fluid entering the interstitium can cause pleural infusion, pericardial effusion and ascites, causing non-cardiogenic pulmonary edema, intestinal edema, muscle edema, oliguria and weight gain. Therefore, patients can present with severe generalized edema and insufficient effective circulating blood volume at the same time. However, the spectrum of CLS associated with CAR-T cell therapy has not been systematically evaluated, which can be a lifethreatening complication. Therefore, as the use of CAR-T therapy continues to expand, it is of great significance to characterize the profile of CLS to help providing better management. A total of 40 ALL patients were included in this study with $11(11 / 40,27.5 \%)$ patients diagnosed as CLS and 29 non-CLS. The baseline characteristics and outcomes of them are described in supplemental materials and Supplementary Table S1.

An analysis of the prevalence of symptoms associated with the diagnosis of CLS has been conducted. Edema, defined as unexplained weight gain $>3 \%$ in $24 \mathrm{~h}$, occurred at 5.1 days postCAR-T infusion in $9(81.8 \%)$ patients with a mean duration of 8.1 days. Nine (81.8\%) CLS patients developed hypotension, where the mean lowest systolic blood pressure was $80 \mathrm{~mm} / \mathrm{Hg}$ and the mean lowest diastolic blood pressure was $48 \mathrm{~mm} / \mathrm{Hg}$. And the average duration of hypotension was 3.5 days. Moreover, the lowest $\mathrm{SpO} 2$ level was lower in CLS patients than in non-CLS patients ( $87.3 \%$ vs. $95.6 \%, p=0.0028)$. For treatment, 7 $(63.6 \%)$ CLS patients used vasopressor and $10(90.9 \%)$ patients used diuretics. Half of the non-CLS patients received gamma globulin infusion while more than $90 \%$ of the CLS patients received it.

In Fig. 1h, the course of disease of the $11 \mathrm{CLS}$ patients in this study was shown as a swimmer plot. It is noted that patient 10 had two periods of CLS, with the first one starting at day 2 and ending at day 11 and the second one starting at day 20 and ending at day 39.

The first fever post-CAR-T infusion occurred at an average of 1.2 days for CLS patients, which is earlier than the 3.4 days for non-CLS patients $(p=0.0206)$. Cytokines associated with the severity of CRS were analyzed in this study. The peak concentration of IL- 6 in CLS patients was much higher than that in non-CLS patients (9059 vs. $1829 \mathrm{pg} / \mathrm{ml}, p=0.001$ ), which is consistent with the well-recognized concept of IL-6 as an indicator of CRS, while the number of days to reach peak IL-6 was not statistically different. The peak IFN- $\gamma$ in CLS patients was more than 4 times higher than that in non-CLS patients(1400 vs. $345 \mathrm{pg} / \mathrm{ml}, p=0.0015)$. As an important CRS indicator, both CLS and non-CLS patients shared similar baseline ferritin pre-CAR-T, while the peak level of ferritin was much higher in CLS patients ( 23340 vs. $8846 \mathrm{pg} / \mathrm{ml}, p=0.0244$ ). Although the number of days to reach peak ferritin post-CAR-T for CLS patients was shorter than that for non-CLS patients, it did not reach statistical significance. It is important to notice that CLS patients had lower levels of serum total protein (TP, 43.8 vs $53.0 \mathrm{~g} / \mathrm{L}, p=0.0006$ ) and serum albumin (ALB, $27.4 \mathrm{vs} 33.9 \mathrm{~g} / \mathrm{L}, p=0.0015)$, while hemoglobin concentrations showed no difference. Other cytokines and laboratory indexes including peak levels of IL-10, peak BNP, number of days to peak BNP, peak c-reactive protein and number of days to reach it showed no significant difference. (Fig. 1b-f).

CLS could be regarded as a syndrome of severe CRS. More than half patients without CLS had $0-2$ grade CRS and the remaining $17.2 \%$ patients had $3-4$ grade CRS. In this study, we found that patients who suffered from CLS all developed 3-4 grade CRS, and patients with high grade of CRS seem to have a greater possibility to develop CLS (Fig. 1g). Patients with CLS develop their first fever post-CAR-T infusion earlier than patients without CLS. (Fig. 1a) For laboratory markers, higher peak IL-6 level, peak IFN- $\gamma$ level, peak ferritin level and lower serum albumin level, total serum protein level after CAR-T infusion are potential markers to develop CLS, although this report is limited by a small sample size. 
a

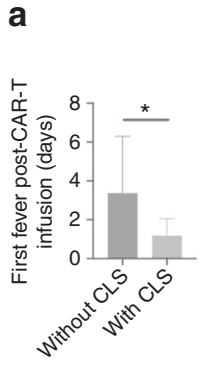

b

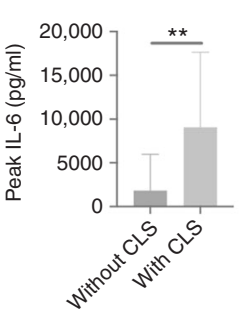

c

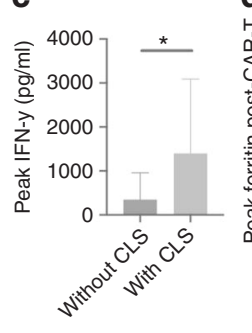

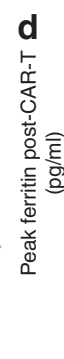

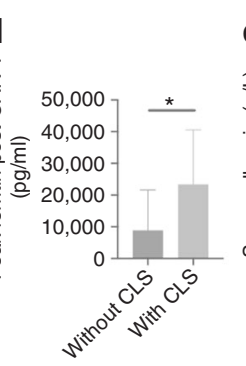

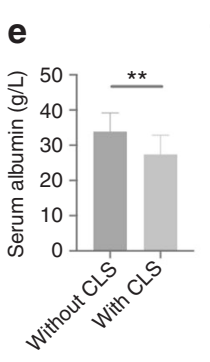

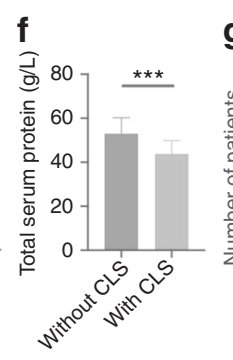

g

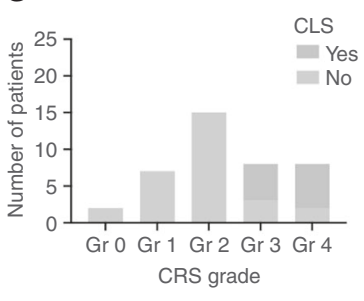

h Patient $1:+$ ro $0+0$ +

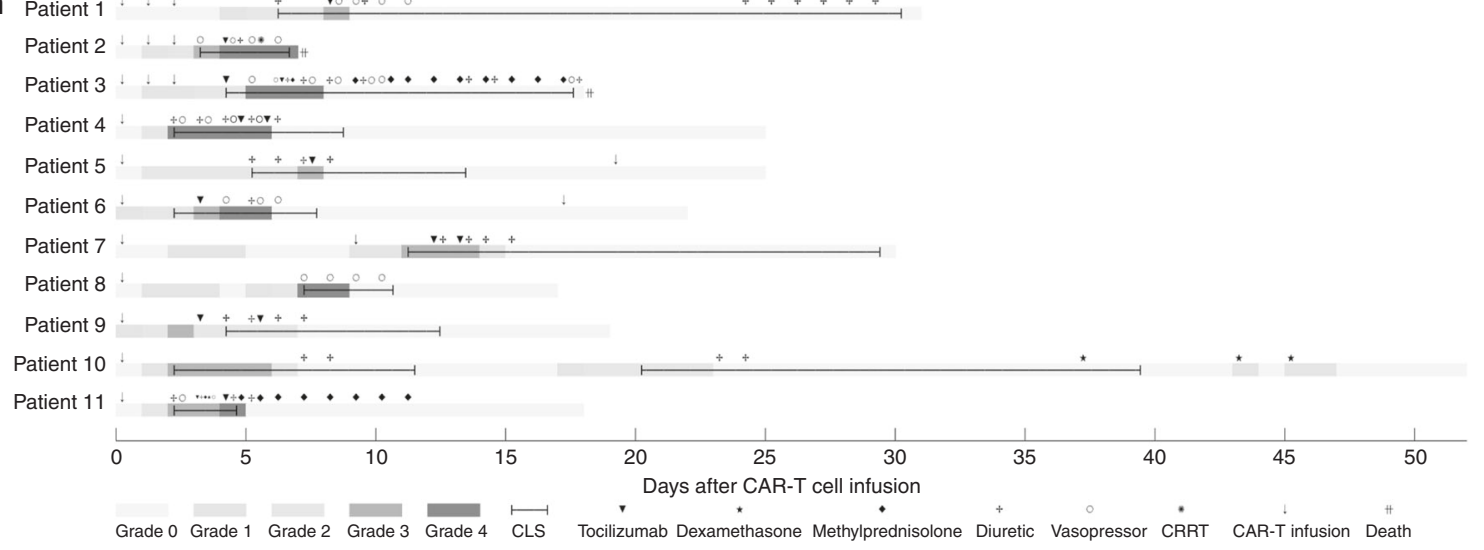

Fig. 1 a The time from the infusion day to the first fever, with patients with CLS experiencing fever symptoms earlier than patients without CLS. b Peak IL-6 concentrations in the blood are higher in patients with CLS than in patients without CLS. c Peak IFN- $\gamma$ concentrations in the blood are higher in patients with CLS than in patients without CLS. $\mathbf{d}$ After the infusion of CAR-T the peak ferritin concentration in the blood of patients with CLS was higher than that of patients without CLS. e The albumin concentration in the blood of patients with CLS was lower than that of patients without CLS. $\mathbf{f}$ The total protein concentration in the blood of patients with CLS was lower than that of patients without CLS. ${ }^{*} p<0.05 ;{ }^{* *} p<0.01 ;{ }^{* * *} p<0.005$. g More than half of the patients with grade 3 or 4 CRS developed CLS and all patients with CLS had grade 3-4 CRS; $\mathbf{h}$ Swimmer plot of presentation, management, and outcomes of patients with CLS. Colors on the swimmer plot indicate the CRS grade on each day from the day of CAR T-cell infusion to the day of discharge in all patients who developed CLS. The duration of CLS and interventions are indicated in the figure. Patient 2 died of CLS and patient 3 died with CLS. Patient 2 used CRRT on day 6. Patient 10 developed 2 times of CLS. Doses of medications: dexamethasone 10 mg intravenously, methylprednisolone $40 \mathrm{mg}$ intravenously, tocilizumab $4-8 \mathrm{mg} / \mathrm{kg}$ intravenously. CLS capillary-leak syndrome, CRRT continuous renal replacement therapy.

CLS includes idiopathic systemic CLS and secondary CLS, characterized by leakage of fluid and macromolecules into tissues. There are other causes of secondary CLS such as COVID-19 infection [7-10]. Post-CAR-T CLS is a complication characterized by severe hypotension, hypoalbuminemia, and hemoconcentration flare-ups, with severe disruption of the vascular endothelium during CLS episodes, resulting in plasma and protein leakage into the interstitial space. The goal of current therapy is to control CRS, reduce inflammatory mediator activation, prevent further capillary leakage, restore blood volume, improve circulatory function, maintain oxygen supply.

The IL-6 receptor antagonist tocilizumab is an important drug for the control of CRS in CAR-T therapy and has not been found to impair the efficacy of CAR-T therapy, so it is possible to control CRS by it and effectively suppress secondary CLS. Steroids can inhibit the inflammatory reaction by inhibiting arachidonic acid metabolism and reducing lysosomal enzyme production, controlling the severity of CRS and improving capillary permeability [11]. At the same time, the application of steroids in CAR-T therapy is controversial $[12,13]$, therefore further research is needed to find a balance of steroids use that controls CLS while preserving CAR-T killing effects. Previous studies have shown that IVIG is an effective treatment for systematic CLS [14]. Some reports of secondary CLS suggest that the earlier the IVIG is used the better the treatment may be $[15,16]$. However, further studies are needed.
Jingjing Feng $\mathbb{D}^{1,2,3,4}$, Mi Shao ${ }^{1,2,3,4}$, Yongxian Hu $\mathbb{D}^{1,2,3,4 凶}$ and
He Huang $\mathbb{D D}^{1,2,3,4 凶}$

${ }^{1}$ Bone Marrow Transplantation Center, the First Affiliated Hospital, Zhejiang University School of Medicine, Hangzhou, China. ${ }^{2}$ Liangzhu Laboratory, Zhejiang University Medical Center, 1369 West Wenyi Road, Hangzhou, China. ${ }^{3}$ Institute of Hematology, Zhejiang

University, Hangzhou, China. ${ }^{4}$ Zhejiang Province Engineering

Laboratory for Stem Cell and Immunity Therapy, Hangzhou, China. Notation of prior abstract publication/presentation: Selected data were presented in abstract form at the 2018 American Society of Hematology meeting. ${ }^{\bowtie e m a i l: 1313016 @ z j u . e d u . c n ; ~}$ huanghe@zju.edu.cn

\section{REFERENCES}

1. Schuster SJ, Bishop MR, Tam CS, Waller EK, Borchmann P, McGuirk JP, et al. Tisagenlecleucel in adult relapsed or refractory diffuse large B-cell lymphoma. $\mathrm{N}$ Engl J Med. 2018;380:45-56.

2. Maude SL, Laetsch TW, Buechner J, Rives S, Boyer M, Bittencourt H, et al. Tisagenlecleucel in children and young adults with B-cell lymphoblastic leukemia. $\mathrm{N}$ Engl J Med. 2018;378:439-48.

3. Park JH, Rivière I, Gonen $M$, Wang $X$, Sénéchal $B$, Curran $K J$, et al. Long-term follow-up of CD19 CAR therapy in acute lymphoblastic leukemia. N Engl J Med. 2018;378:449-59.

4. Gutgarts V, Jain T, Zheng J, Maloy MA, Ruiz JD, Pennisi M, et al. Acute kidney injury after CAR-T cell therapy: low incidence and rapid recovery. Biol Blood Marrow Transplant. 2020. 
5. Burstein DS, Maude S, Grupp S, Griffis H, Rossano J, Lin K. Cardiac profile of chimeric antigen receptor $\mathrm{T}$ cell therapy in children: a single-institution experience. Biol Blood Marrow Transpl. 2018;24:1590-5.

6. Lee DW, Santomasso BD, Locke FL, Ghobadi A, Turtle CJ, Brudno JN, et al. ASTCT consensus grading for cytokine release syndrome and neurologic toxicity associated with immune effector cells. Biol Blood Marrow Transpl. 2019;25:625-38.

7. Case R, Ramaniuk A, Martin P, Simpson PJ, Harden C, Ataya A. Systemic capillary leak syndrome secondary to coronavirus disease 2019. Chest. 2020;158:e267-e8

8. de Chambrun MP, Cohen-Aubart F, Donker DW, Cariou P-L, Luyt C-E, Combes A, et al. SARS-CoV-2-induces acute and refractory relapse of systemic capillary leak syndrome (Clarkson's disease). Am J Med. 2020.

9. de Chambrun MP, Constantin J-M, Mathian A, Quemeneur C, Lepere V, Combes A, et al. Clarkson's disease episode or secondary systemic capillary leak-syndrome: that is the question! Chest. 2021;159:441.

10. Wu MA, Fossali T, Pandolfi L, Carsana L, Ottolina D, Frangipane V, et al. COVID-19: The key role of pulmonary capillary leakage. An observational cohort study. medRxiv. 2020. https://www.medrxiv.org/content/10.1101/2020.05.17.20104877v1.

11. Casadei Gardini A, Aquilina M, Oboldi D, Lucchesi A, Carloni S, Tenti E, et al. Separate episodes of capillary leak syndrome and pulmonary hypertension after adjuvant gemcitabine and three years later after nab-paclitaxel for metastatic disease. BMC Cancer. 2013;13:542.

12. Strati $P$, Ahmed S, Furqan F, Fayad LE, Lee HJ, lyer SP, et al. Prognostic impact of corticosteroids on efficacy of chimeric antigen receptor T-cell therapy in large B-cell lymphoma. Blood. 2021;137:3272-6.

13. Liu S, Deng B, Pan J, Yin Z, Lin Y, Ling Z, et al. Corticosteroids do not influence the efficacy and kinetics of CAR-T cells for B-cell acute lymphoblastic leukemia. Blood. 2019;134:228

14. Xie Z, Chan EC, Long LM, Nelson C, Druey KM. High-dose intravenous immunoglobulin therapy for systemic capillary leak syndrome (Clarkson disease). Am J Med. 2015;128:91-5.

15. Prete M, Urso L, Fatone MC, Pinto V, Perosa F. Antiphospholipids syndrome complicated by a systemic capillary leak-like syndrome treated with steroids and intravenous immunoglobulins: a case report. Medicine. 2016;95:e2648.

16. Natterer J, Perez MH, Di Bernardo S. Capillary leak leading to shock in Kawasaki disease without myocardial dysfunction. Cardiol Young. 2012;22:349-52.

\section{AUTHOR CONTRIBUTIONS}

JF collected the data and designed and write the article. MS collected the data. YH and $\mathrm{HH}$ designed the research and reviewed the article.

\section{FUNDING}

This work was supported by the grants from 973 Program (2015CB964900), the Natural Science Foundation of China (82000179), Key Project of Science and Technology Department of Zhejiang Province (2018C03016-2, 2015C03G2150011), Zhejiang public welfare foundation (GF18H180002).

\section{COMPETING INTERESTS}

The authors declare no competing interests.

\section{ADDITIONAL INFORMATION}

Supplementary information The online version contains supplementary material available at https://doi.org/10.1038/s41409-022-01562-4.

Correspondence and requests for materials should be addressed to Yongxian Hu or He Huang.

Reprints and permission information is available at http://www.nature.com/ reprints

Publisher's note Springer Nature remains neutral with regard to jurisdictional claims in published maps and institutional affiliations.

Notation of prior abstract publication/presentation Selected data were presented in abstract form at the 2018 American Society of Hematology meeting 